\title{
Supporting Information for \\ Plasmonic Metamaterial Gels with Spatially Patterned Orientational Order via 3D Printing
}

Andrew J. Hess ${ }^{\dagger}$, Andrew J. Funk ${ }^{\dagger}$, Qingkun Liu ${ }^{\dagger *}$, Joshua A. De La Cruz ${ }^{\star}$, Ghadah H. Sheetah $^{{ }^{\perp}}$, Blaise Fleury ${ }^{\dagger}$, and Ivan I. Smalyukh ${ }^{\dagger \& *}$

${ }^{\dagger}$ Department of Physics, 390 UCB, University of Colorado Boulder, Boulder, CO 80309, USA

Materials Science and Engineering, $027 \mathrm{UCB}$, Sustainability, Energy \& Environment

Community, University of Colorado Boulder, Boulder, CO 80303, USA

${ }^{\S}$ Renewable and Sustainable Energy Institute, National Renewable Energy Laboratory and University of Colorado, Boulder, CO 80309, USA

Table of Contents

1. Preparation of Gold Nanorods

2. Preparation of Cellulose Nanocrystals

3. Preparation of Hydrogels: Procedure 1

4. Preparation of Hydrogels: Procedure 2

5. 3D Printing Equipment Design

6. 3D Printing Procedures and Parameters

7. Preparation of Aerogels

8. Optical Imaging and Spectroscopy

9. Scalar Order Parameter of Plasmonic Inclusions, Spectral Data Collection, and Analysis Methodology

10.Electron Microscopy 


\section{Preparation of Gold Nanorods (GNRs)}

We synthesized the GNRs using a seed-mediated method. ${ }^{[\mathrm{S} 1]}$ To prepare the seed solution, 0.1 $\mathrm{mL}$ of an aqueous $0.025 \mathrm{M}$ solution of chloroauric acid $\left(\mathrm{HAuCl}_{4} \cdot 3 \mathrm{H}_{2} \mathrm{O}\right.$, Sigma-Aldrich) was added to $10 \mathrm{~mL}$ of a $0.10 \mathrm{M}$ cetyl trimethyl ammonium bromide (CTAB, Sigma-Aldrich) solution in a conical flask. The solution changed its color from clear to brown-yellow, indicating the formation of gold seeds, when $0.6 \mathrm{~mL}$ of an aqueous $0.01 \mathrm{M}$ ice-cold sodium borohydride (Sigma-Aldrich) solution was added by vigorous stirring. Separately, the growth solution was prepared by mixing $5 \mathrm{~mL}$ of deionized (DI) water, $5 \mathrm{~mL}$ of $0.20 \mathrm{M} \mathrm{CTAB}, 0.200 \mathrm{~mL}$ of $0.025 \mathrm{M}$ $\mathrm{HAuCl}_{4} \cdot 3 \mathrm{H}_{2} \mathrm{O}$, and $0.040 \mathrm{~mL}$ of $0.016 \mathrm{M}$ silver nitrate solutions in a conical flask. Then 0.090 $\mathrm{mL}$ of $0.08 \mathrm{M}$ ascorbic acid was added to the growth solution. Finally, $0.012 \mathrm{~mL}$ of seed solution was added to the growth solution. The combined solution was undisturbed at $27{ }^{\circ} \mathrm{C}$ for at least $10 \mathrm{~h}$. Subsequently, it was centrifuged at $7.8 \mathrm{kRCF}$ for $30 \mathrm{~min}$. twice and re-dispersed in DI water to remove excess CTAB. Next, the GNRs were surface-functionalized by thiol-terminated methoxy-poly(ethylene glycol) (mPEG-SH, JemKem Technology) to minimize aggregations of the GNRs within aqueous lyotropic dispersions of CNCs in a sol-phase material. ${ }^{[2]}$ For this, 1 $\mathrm{mL}$ of aqueous solution with $30 \mathrm{mg}$ of $5 \mathrm{kDa}$ mPEG-SH was added to $50 \mathrm{~mL}$ of a diluted GNR dispersion with an optical density of 4 . After standing for $24 \mathrm{~h}$, this dispersion was purified via centrifugation to remove the excess mPEG-SH. The GNRs were then washed three times via centrifugation at $7.8 \mathrm{kRCF}$ for $20 \mathrm{~min}$. and redispersed into DI water for further usage.

\section{Preparation of Cellulose Nanocrystals (CNCs)}

Cellulose nanocrystals were synthesized via sulfuric-acid catalyzed hydrolysis of cotton balls as described elsewhere, ${ }^{[\mathrm{S} 2]}$ albeit with slight modifications. Briefly, cellulose (Swisspers brand cotton balls) was reacted as-is with sulfuric acid $\left(\mathrm{H}_{2} \mathrm{SO}_{4}\right.$, ACS Plus, Fisher chemical $)$ at a ratio of 
$1 \mathrm{~g}$ of cellulose to $5 \mathrm{~mL}$ of sulfuric acid to $5 \mathrm{~mL}$ of DI water in a reaction flask placed inside a sonicator (Branson 3800 ), whose bath temperature was held at $46-48^{\circ} \mathrm{C}$. The reaction was mechanically stirred; the stirring speed ( 700-1200 rpm) was adjusted every $15 \mathrm{~min}$. to be as fast as possible while minimizing splashing on the vessel walls. The reaction proceeded for $2 \mathrm{~h}$ under continuous sonication and was subsequently quenched by threefold dilution with DI water while in an ice bath. The reaction fluid was then repeatedly washed via centrifugation at 10-11 kRCF until a turbid solution was obtained, typically after $4-5$ wash cycles. Afterward, any gelatinous subnatant was discarded and the turbid supernatant was dialyzed against a cellulose membrane with 12,000-14,000 molecular weight cutoff. The dialysis water bath was changed every few hours until its $\mathrm{pH}$ remained at 7 for at least $6 \mathrm{~h}$. Then the aqueous $\mathrm{CNCs}$ were collected, bath sonicated for $5 \mathrm{~min}$., and then centrifuged one last time at $5 \mathrm{kRCF}$ for $5 \mathrm{~min}$., to remove any remaining dehydrated cellulose. The supernatant was filtered against a cellulose nitrate membrane with an average pore size of $5 \mu \mathrm{m}$ to obtain purified aqueous CNCs with a concentration $<1 \mathrm{wt} \%$. Finally, the CNCs were concentrated in a loosely covered glass dish on a hotplate with a surface temperature of $30^{\circ} \mathrm{C}$ with moderate stirring until the desired concentration was obtained. The $\mathrm{CNCs}$ were then stored in sealed glass jars in a $4-^{\circ} \mathrm{C}$ refrigerator until needed.

\section{Preparation of Hydrogels: Procedure 1}

Approximately $5 \mathrm{wt} \%$ acrylamide monomers (AAm, Acros Organics) were added to an aqueous CNC dispersion whose initial concentration was $28.2 \mathrm{wt} \%$. The CNC dispersion comprised $\sim 76$ wt $\%$ of the final sol before printing. The solution was vigorously stirred and bath sonicated to thoroughly mix the AAm. Next, while vigorously stirring, the solution's $\mathrm{pH}$ was adjusted to neutral or basic by the dropwise addition of an aqueous sodium hydroxide (Fisher Chemical) 
solution. An aqueous GNR colloidal dispersion was added to bring the GNR's final concentration to $\sim 0.023 \mathrm{wt} \%$. The AAm-CNC-GNR dispersion was mixed thoroughly using a vortex mixer. Approximately $0.1 \mathrm{wt} \%$ of the photo-initiator 1-[4-(2-Hydroxyethoxy)-phenyl]-2hydroxy-2-methyl-1-propane-1-one (Irgacure-2959, Sigma Aldrich) and 0.06 wt\% of base catalyst N,N,N',N'-tetramethylethane-1,2-diamine (TEMED, Acros Organics) were directly added to the dispersion. Both the Irgacure-2959 and TEMED were vigorously mixed into the AAm-CNC-GNR dispersion by a vortex mixer. Finally, N,N'-methylenebis(acrylamide) (MBAA, Acros Organics), which was used as a cross-linker, was separately dissolved in DI water. An aliquot was taken and mixed by vortexing with the dispersion to bring the MBAA's final concentration to $\sim 0.04 \mathrm{wt} \%$. DI water was added to bring the total to $100 \%$. Immediately after the AAm-CNC-GNR sol was prepared, it was stored in a $4-{ }^{\circ} \mathrm{C}$ refrigerator for up to two days prior to printing.

\section{Preparation of Hydrogels: Procedure 2}

Approximately $20 \mathrm{wt} \%$ or $\sim 9 \mathrm{wt} \%$ AAm were added to a $\sim 63 \mathrm{wt} \%$ or $\sim 73 \mathrm{wt} \%$ aqueous CNC dispersion whose initial CNC concentration was $\sim 1 \mathrm{wt} \%$ or $\sim 14 \mathrm{wt} \%$ for a total CNC concentration of $\sim 0.8 \mathrm{wt} \%$ or $\sim 10 \mathrm{wt} \%$ of the final sol, respectively. The solution was vigorously stirred and bath sonicated to thoroughly mix the AAm. Next, while vigorously stirring, the solution's $\mathrm{pH}$ was adjusted to neutral or basic by the dropwise addition of aqueous sodium hydroxide solution. An aqueous GNR colloidal dispersion was added to bring the GNR's final concentration to $\sim 0.023 \mathrm{wt} \%$. The AAm-CNC-GNR dispersion was mixed thoroughly using a vortex mixer. Approximately $0.1 \mathrm{wt} \%$ of the photo-initiator Irgacure-2959 and $\sim 0.06 \mathrm{wt} \%$ of the base-catalyst TEMED were directly added to the dispersion. Both the Irgacure-2959 and TEMED were vigorously mixed into the AAm-CNC-GNR dispersion by a vortex mixer. The 
dispersion, while inside a 20 -mL glass scintillation vial, was exposed to UV light $\left(\sim 2 \mathrm{~mW} \mathrm{~cm}^{-2}\right.$ at $\sim 275 \mathrm{~nm})$ for $\sim 50 \mathrm{~min}$. ( $\sim 0.8 \mathrm{wt} \% \mathrm{CNCs})$ or $\sim 40 \mathrm{~min}$. ( $\sim 10 \mathrm{wt} \% \mathrm{CNCs})$ while slowly stirred for $3 \mathrm{~min}$. and rested for $3 \mathrm{~min}$. repeatedly.

The UV-exposure time was adjusted to yield a sol with a viscosity within the ideal working range for our direct-ink-writing (DIW) printer. A qualitative viscosity test was developed to determine a suitable range of viscosities for printed sols. The sol's scintillation vial was rotated upside-down and observed for $\sim 1 \mathrm{~min}$. If negligible flow was visibly detected and if, upon insertion into the gel and subsequent retraction of a spatula, the spatula drew a bead of material similar in elasticity and texture to saliva, then the sol would be printed. Otherwise, the UV-exposure duration was increased and implemented in the described manner above.

Next, MBAA was separately dissolved in DI water. An aliquot was taken and mixed by vortexing with the oligomerized, viscous dispersion to bring the MBAA's final concentration to $\sim 0.04 \mathrm{wt} \%$. DI water was added to bring the total to $100 \%$. Immediately after the PAM-CNCGNR sol was prepared, it was stored in a $4-{ }^{\circ} \mathrm{C}$ refrigerator for up to two days prior to printing.

\section{3D Printing Equipment Design}

A fused-filament-fabrication (FFF) 3D printer (Aleph Objects, Inc. Lulzbot Mini v1) was retooled in-house to print as a DIW printer. Briefly, a syringe (5-60 mL, Figure S1(a) item i) was inserted into a syringe pump (Figure S1(a) item ii, Yale Apparatus Multi-Phaser Model YA-12). A length of flexible vinyl tubing (Figure S1(a) item iii) was installed between (i) and another syringe with its plunger cut off (Figure S1(a) item iv) so that (iv) was actuated hydraulically from the rear. The closed volume defined by (i), (iii), and (iv) was completely filled with water. Sol was then introduced through (iv)'s orifice and extruded through a separate length of stiff tubing in place of a needle (Figure S1(a) item v). The syringe and tube assembly (Figure S1(b) 
item iv and v) guided sol to the printer's extrusion nozzle (Figure S1(b) item vi, Lulzbot Mini Tool Head v2) and replaced the plastic filament typically used for this printer. The entire assembly is presented in Figure S1(c) with a schematic representation in Figure S1(d). Sol was extruded from (vi) onto the printing substrate (Figure S1(c) or (d) item vii). In our case, we printed directly on microscope slide glass, shown in Figure S1(c) affixed atop item (vii), instead of atop the reddish stock printer substrate (vii). 3D motion was achieved along the coordinate axes shown (Figure S1(d)) by translating the print head (Figure S2(b)) along x and z and separately translating the printing substrate (vii) along y.
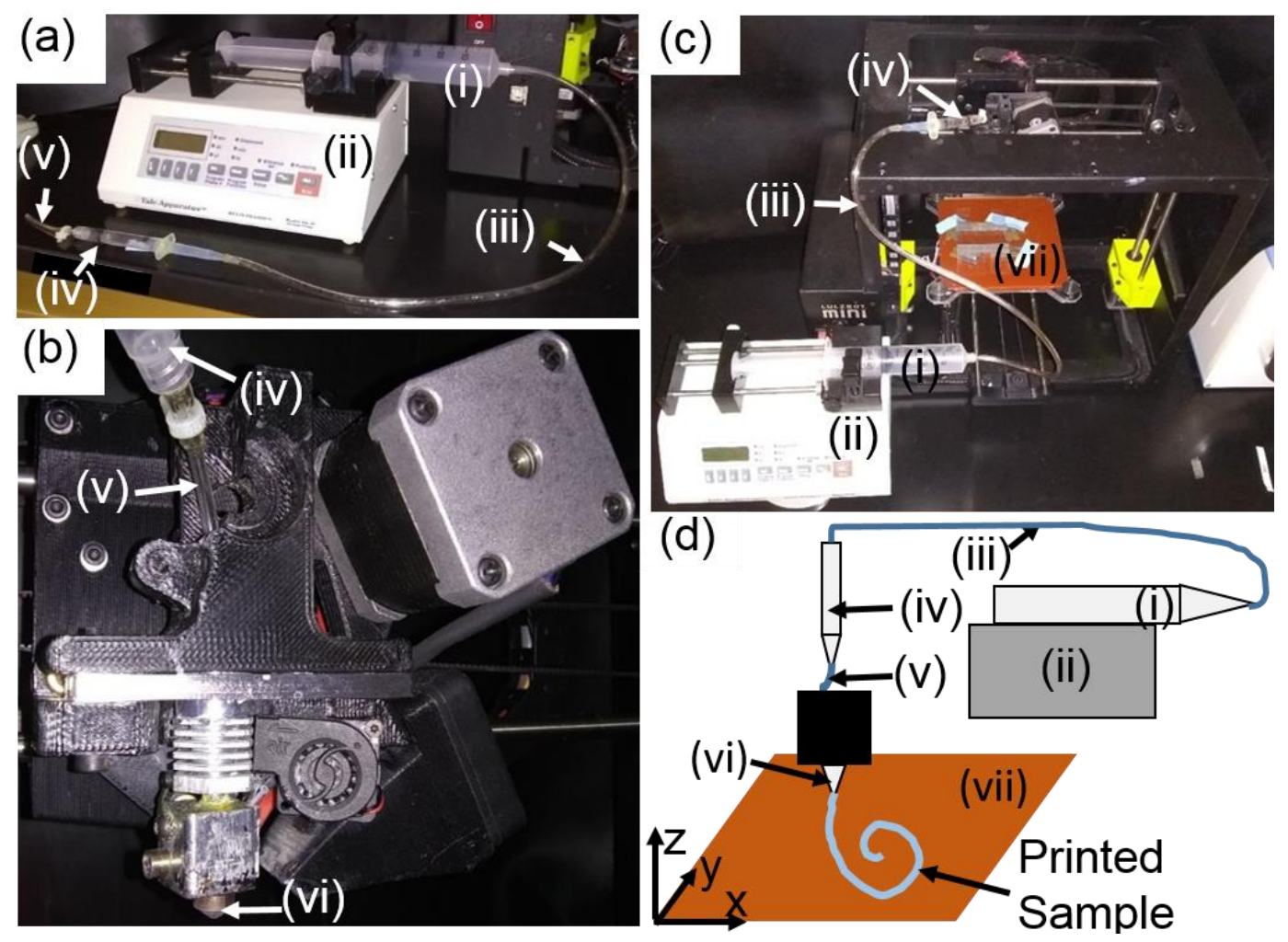

Figure S1. (a) The hydraulic system enabling extrusion of gelatinous sol from the 3D printer. (b) The modified print head with a syringe and tube (item iv and v) conveying sol to the extrusion nozzle (item vi). (c) The completely assembled 3D printer. (d) A schematic representation of the $3 \mathrm{D}$ printing system shown in (c). 


\section{3D Printing Procedures and Parameters}

Sol material was warmed to room temperature and introduced through the second syringe's orifice (Figure S1(a) item iv and v) by running the syringe pump in reverse. Typically, a total volume of $\sim 3 \mathrm{~mL}$ of sol would be used for each printing batch. The second syringe replaced the tool head's original sprocket assembly. A new short length of stiff yet flexible tubing connected the second syringe's orifice to the tool head's hot-end filament heater and extrusion nozzle by inserting the stiff tubing in place of typical FFF filament (Figure S1(b)).

Printed objects were designed with Python code formatted according to the g-code commands used by the printer's controlling and slicing software (Matter Control v.1.7.). The gcode file contained the starting and ending sequences as well as the code for the printed object. Care was taken to modify the starting sequence to operate the printer's extrusion nozzle and printing surface at room temperature, to override the printer's low-temperature extrusion exception, and to account for the modified starting height due to the printing substrate.

The extrusion nozzle was primed with the sol by briefly running the syringe pump at an elevated rate of $\sim 0.6 \mathrm{~mL} \mathrm{~min}^{-1}$. Once the sol was visibly detected at the tip of the nozzle, the syringe pump was stopped. Excess sol was wiped away. Next, the printer's starting sequence was activated. Tens of seconds before the printer's starting sequence was completed, the syringe pump was re-started at the extrusion rate. The sol filament was printed on a microscope-slideglass substrate. A pump rate of $\sim 0.06 \mathrm{~mL} \mathrm{~min}^{-1}$, a tool-head and print-surface raster speed of 95 $\mathrm{mm} \mathrm{min}^{-1}$, and a nozzle height of $\sim 0.35 \mathrm{~mm}$ from the substrate for a 0.5 -mm-diameter nozzle enabled the printing of uniform, contiguous, and aesthetically appealing shapes. Immediately after printing, the sample was exposed to UV radiation for cross-linking. For Irgacure-2959's peak extinction at $\sim 275 \mathrm{~nm}$, the sol was exposed to an intensity of $\sim 2 \mathrm{~mW} \mathrm{~cm}{ }^{-2}$ for $5-12 \mathrm{~min}$. 
atop a black light (ADJ UV Panel HP with four bulbs LL-UVP40) or to an intensity of $\sim 82 \mathrm{~mW}$ $\mathrm{cm}^{-2}$ for up to $1 \mathrm{~min}$. from a UV-curing lamp (Omnicure Series 2000, Lumen Dynamics). The hydrogel was placed in a Petri dish with a few water drops to prevent the hydrogel's dehydration during storage. The Petri dish was then sealed immediately after curing.

\section{Preparation of Aerogels}

To convert a hydrogel into an aerogel, the hydrogel was first converted into the intermediary alcogel state by three times immersion in $20 \mathrm{~mL}$ of absolute ethanol for at least $12 \mathrm{~h}$ each rinse. Alcogels were then converted to aerogels via carbon dioxide critical point dryer (CPD) (Automegasamdri 915B or Samdri-780A, Tousimis, Inc.). Before the alcogel was inserted into the CPD chamber, the chamber was filled approximately half full with ethanol. The alcogel and its attached glass microscope slide were then placed in the chamber. Liquid carbon dioxide used for solvent exchanges was pressurized at $5.5 \mathrm{MPa}$ and purged from the chamber until all ethanol was removed. Next, the chamber was heated beyond carbon dioxide's critical point to $\sim 40{ }^{\circ} \mathrm{C}$ and $\sim 8.3 \mathrm{MPa}$. After the critical point was reached, it was held for $\sim 15$ min. to allow any fluid trapped within the gel to become supercritical. Finally, while maintaining the temperature at $40{ }^{\circ} \mathrm{C}$, the pressure was bled off at a maximum rate of $\sim 0.34 \mathrm{MPa} \min ^{-1}$ until the chamber pressure reached 1.7 MPa, after which the carbon dioxide gas was exhausted as quickly as possible. After the aerogel was removed from the chamber, it was sealed and stored at a relative humidity of $\sim 20 \%$ at room temperature.

\section{Optical Imaging and Spectroscopy}

Optical micrographs were obtained using an Olympus BX-51 microscope with $2 \times$ and $4 \times$ magnification. Transmission-mode brightfield microscopy and polarizing optical microscopy (POM) with and without a $530 \mathrm{~nm}$ full-wave retardation plate were used to qualitatively 
determine the gels' director and thickness variation. Optical micrographs were captured with Diagnostic Instruments, Inc. Spot Insight 14.2 Color Mosaic CCD camera. Other optical images were captured with a Nikon D300 Digital Camera with a Nikon AF-S Nikkor 18 - 200 mm lens. Spectra were obtained in transmission mode from the microscope's sample plane and with a fiber-optic adapter affixed in place of the microscope's camera. The sample's extinction spectra were recorded via an Ocean Optics USB2000-FLG spectrometer.

\section{Degree of Order of Plasmonic Inclusions, Spectral Data Collection, and Analysis}

\section{Methodology}

From previous results, which used static methods to co-align GNRs and CNCs, ${ }^{[\mathrm{S} 3]}$ we were motivated to understand the attainable degree of alignment for GNRs through DIW 3D printing methods. The extent of anisotropic GNRs' average angular fluctuations away from a uniform director field $N$ can be quantified with a scalar order parameter $S$, where $S=0$ represents no orientational ordering and $S=1$ indicates perfect orientational alignment along $N$. Measured longitudinal surface plasmon resonance (LSPR) and transverse surface plasmon resonance (TSPR) extinction spectra were used to calculate the GNRs' scalar order parameters in Procedure 1 and Procedure 2, where $S_{1}=0.45 \pm 0.13$ and $S_{2}=0.34 \pm 0.07$, respectively. Representative TSPR and LSPR extinction spectra for PAM-CNC-GNR gels with $21.5 \mathrm{wt} \%$ CNCs, prepared according to Procedure 1, and for gels with $10.2 \mathrm{wt} \%$ CNCs, prepared according to Procedure 2, are shown in Figure S2(a). For gels with $0.76 \mathrm{wt} \% \mathrm{CNCs}$, prepared according to Procedure 2, no observable polarization-dependent extinction peak was evident, thereby indicating isotropic alignment of GNRs with $S=0$. A representative extinction spectrum taken with unpolarized incident light is shown in Figure S2(b). In this case, rather than the emergence of two distinct TSPR and LSPR extinction peaks, instead a single, broadened extinction peak is observed. The 
existence of such an extinction profile is likely indicative of complex, coupled configurations of GNRs within the PAM-CNC-GNR gel. ${ }^{[\mathrm{S} 4]}$ A possible alignment of CNCs and GNRs in the 21.5wt\% and 10.2-wt\% gels is depicted in Figure S2(a)'s inset while an isotropic organization of the 0.76-wt\%-PAM-CNC-GNR gel is represented by the inset in Figure S2(b).
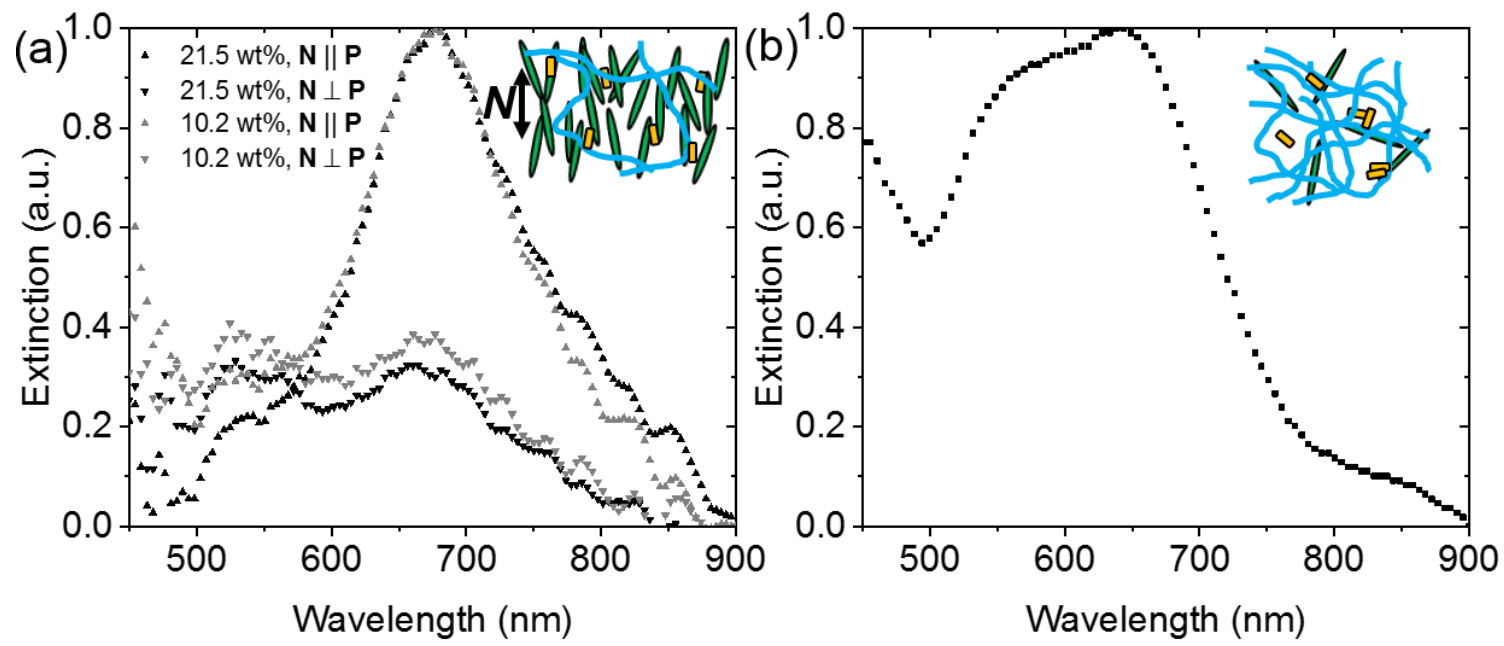

Figure S2. Spectral characterization of PAM-CNC-GNR hydrogel LSPR $(\boldsymbol{N} \| \boldsymbol{P})$ and TSPR $(\boldsymbol{N} \perp$ $\boldsymbol{P}$ ) extinction modes with insets demonstrating possible alignment of CNCs and GNRs. Crosslink symbols are omitted for clarity. (a) Spectral comparisons between representative extinction curves from hydrogels printed according to Procedure 1 (21.5 wt\% CNCs) and Procedure 2 (10.2 wt\% CNCs). Orientational co-alignment of GNRs and CNCs, indicated by director $N$ with the double headed arrow, (inset) is crucial for polarization selective metamaterials. (b) A representative polarization-independent extinction spectrum from a hydrogel (0.76 wt\% CNCs) with isotropic GNR and CNC organization (inset) prepared with Procedure 2.

The extinction spectra corresponding to the 10.2-wt\%-CNC data shown in Figure S2(a) were chosen from a larger set, which is displayed in Figure S3(a). The 21.5-wt\%-CNC data shown as well in Figure S2(a) originate from the set shown in Figure S3(b). For the data in Figure S3(a) and (b), two spectra corresponding to each sample's LSPR $(\boldsymbol{N} \| \boldsymbol{P})$ and TSPR $(\boldsymbol{N} \perp$ $\boldsymbol{P})$ were collected for each manufactured sample. The LSPR spectra are shown in black while those for the TSPRs are displayed in grey. All spectra were normalized to zero at $900 \mathrm{~nm}$. Next, the LSPR extinction spectra were scaled by a scaling coefficient to unity at their extinction peaks. 
For each pair of LSPR and TSPR spectra, the TSPR spectrum in a pair was scaled by the corresponding LSPR scaling coefficient. This scaling approach was used because of sample thickness and illumination intensity differences among the collected spectral data. To determine the GNRs' scalar order parameter $S_{G N R}$, the extinction formula was used: $S_{G N R}=\frac{A_{\|}-A_{\perp}}{A_{\|}+2 A_{\perp}}$, where $A_{\|}$and $A_{\perp}$ represent the magnitudes of the longitudinal extinction peaks for $\boldsymbol{P}_{\|} \| \boldsymbol{N}$ and $\boldsymbol{P}_{\perp} \perp \boldsymbol{N}$, respectively. ${ }^{[\mathrm{S} 3]}$ For the first method used to determine $S_{G N R}$, the scalar order parameter was calculated for each spectral pair without scaling. Next, the mean and standard deviation of the scalar order parameters were calculated to estimate total uncertainty. As the second method, the means and standard deviations for both $A_{\|}$and $A_{\perp}$ were calculated from the populations shown in Figure S3(a) and (b). A standard combined uncertainty calculation yielded $\delta S_{G N R}=$ $\frac{3}{\left(\delta A_{\|}+2 \delta A_{\perp}\right)^{2}} \sqrt{\left(\delta A_{\|} A_{\perp}\right)^{2}+\left(A_{\|} \delta A_{\perp}\right)^{2}}$. Of the two methods, the larger values are presented above. Calculation of the dichroic ratios for both Procedure 1 and 2 used the normalized curves shown in Figure S3(a) and (b). The means and standard deviations for both $A_{\|}$and $A_{\perp}$ were used to calculate the mean dichroic ratio $R=\frac{A_{\|}}{A_{\perp}}$ and the standard combined uncertainty $\delta R_{G N R}=$ $\sqrt{\left(\frac{\delta A_{\|}}{A_{\perp}}\right)^{2}+\left(\frac{A_{\|} \delta A_{\perp}}{A_{\perp}{ }^{2}}\right)^{2}}$. The dichroic ratios for Procedure 1 and 2 were found to be $R_{1}=3.2 \pm 1.2$ and $R_{2}=2.6 \pm 0.46$.

Finally, the representative spectrum from those in Figure S3(c) is shown in Figure S2(b). Because the GNRs are isotropic, $S_{G N R}=0$. Thus, unpolarized incident light was used to collect the spectra shown in Figure S3(c). Each curve has been normalized to zero at $900 \mathrm{~nm}$ and scaled to unity at the extinction peak near $650 \mathrm{~nm}$. One spectrum per sample was taken. 

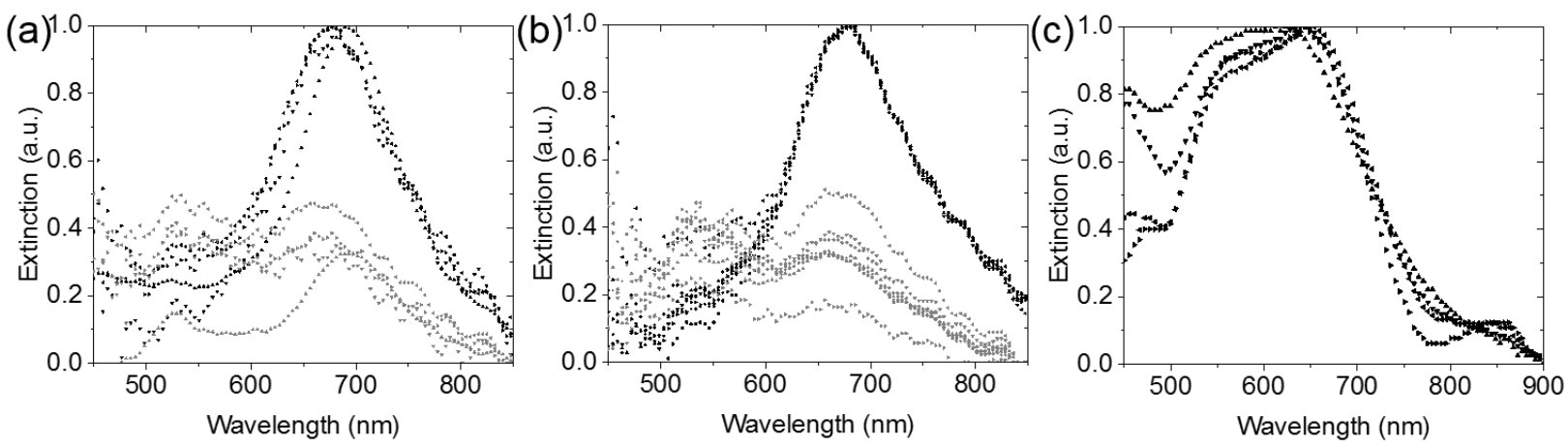

Figure S3. (a, b) LSPR (black) and TSPR (grey) spectra used to determine the GNRs' order parameter within hydrogels containing $10.2 \mathrm{wt} \%$ CNCs (a) and $21.5 \mathrm{wt} \%$ CNCs (b). (c) Polarization-independent extinction spectra of GNRs within hydrogels containing $0.76 \mathrm{wt} \%$ CNCs.

\section{Electron Microscopy}

Transmission electron microscopy (TEM) micrographs of gold nanorods (GNRs) were obtained with a FEI Tecnai T12 Spirit at $100 \mathrm{kV}$ accelerating voltage and at $13000-68000 \times$ magnification. Micrographs of aqueous GNRs were obtained by dropcasting and subsequently drying 0.00028 wt\% GNRs on Cu Formvar Square 300 Mesh grids. Focused ion beam and scanning electron microscopy (FIB-SEM) cross-section imaging was performed using a FEI Nova 600 nanolab dualbeam. The aerogel sample was mounted on a regular SEM stub. For Figure 1(d), the sample was placed carefully inside the microscope, with the top of the sample tilted by $52^{\circ}$ to face directly the FIB. After protecting with a band of $\mathrm{Pt}$, a trench $\sim 5 \mu \mathrm{m}$ wide by $3 \mu \mathrm{m}$ long by $\sim 1 \mu \mathrm{m}$ deep was milled using the ion beam at $30 \mathrm{kV}$ and $93 \mathrm{pA}$. After milling the sample, a thin layer of $\mathrm{Pt}$ was deposited in situ on the fresh surface. SEM images were taken at $5 \mathrm{kV}$ accelerating voltage, $15000 \times$ magnification, and $5.1 \mathrm{~mm}$ WD using the through-the-lens detector (TLD) with secondary-electron detection. To ensure no astigmatism in the image, circular features of the gel were imaged with different sources and detectors. No astigmatism was detected for the electron source with the secondary-electron TLD. For Figure 1(e), the sample was tilted by $52^{\circ}$ to face directly the FIB. After protecting with a band of Pt, a trench $\sim 5$ 
$\mu \mathrm{m}$ wide by $3 \mu \mathrm{m}$ long by $1.7 \mu \mathrm{m}$ deep was milled using the ion beam at $30 \mathrm{kV}$ and $93 \mathrm{pA}$. After milling the sample, a thin layer of Pt was deposited in situ on the fresh cross-section. SEM images were taken at $5 \mathrm{kV}$ accelerating voltage, $25000 \times$ magnification, and $5.1 \mathrm{~mm}$ WD using the TLD with secondary-electron detection.

\section{References}

[S1] J. Perez-Juste, L. M. Liz-Marzan, S. Carnie, D. Y. C. Chan, P. Mulvaney, "Electric-FieldDirected Growth of Gold Nanorods in Aqueous Surfactant Solutions." Adv. Funct. Mater. 2004, 14,571

[S2] J. A. De La Cruz, Q. Liu, B. Senyuk, A. W. Frazier, K. Peddireddy, I. I. Smalyukh, "Cellulose-Based Reflective Liquid Crystal Films as Optical Filters and Solar Gain Regulators." ACS Photonics 2018, 5, 2468

[S3] Q. Liu, M. G. Campbell, J. S. Evans, I. I. Smalyukh, “Orientationally Ordered Colloidal Co-Dispersions of Gold Nanorods and Cellulose Nanocrystals." Adv. Mater. 2014, 26, 7178

[S4] A. E. Ershov, A. P. Gavrilyuk, S. V. Karpov, P. N. Semina, "Effect of Local Environment in Resonant Domains of Polydisperse Plasmonic Nanoparticle Aggregates on Optodynamic Processes in Pulsed Laser Fields." Chin. Phys. B 2015, 24, 047804 